\title{
DESPERTANDO VOCACIONES TIC MEDIANTE JUEGOS Y ANIMACIONES
}

Gladys Dapozo, Cristina Greiner, Gabriel Pedrozo Petrazzini, Jorge Chiapello (*)

\section{Resumen}

La formación universitaria de profesionales de la Informática presenta en la actualidad la siguiente problemática: decrece el interés de los jóvenes en seguir carreras vinculadas con la disciplina, y este desinterés es más marcado en las mujeres. Este fenómeno tiene alcance mundial, y desde las políticas públicas se implementan distintas medidas para revertir esta situación. Desde el año 2013, la Facultad de Ciencias Exactas participa activamente en el programa Vocaciones en TIC, impulsado por la Fundación Sadosky, mediante la realización de talleres para desarrollar juegos y animaciones destinados a los alumnos del nivel medio. En este trabajo se describe la experiencia de las visitas a las escuelas de la ciudad de Corrientes y se muestran los resultados obtenidos de una encuesta orientada a identificar el perfil tecnológico de los alumnos, su interés por seguir carreras vinculadas con la Informática y los factores que influyen en la elección. Los resultados sugieren que motivar tempranamente a los alumnos del nivel medio en el conocimiento práctico de las tecnologías informáticas incrementará su interés por seguir carreras vinculadas con la Informática.

Palabras clave: Articulación universidad-nivel medio. Carreras TIC. Tecnologías informáticas.

\section{Introducción}

Es indudable que en la actualidad las TIC (Tecnología de la Información y la Comunicación) marcan un sendero transversal, liderando el proceso de transformación tecnológica a través de sus efectos sinérgicos sobre los distintos sectores sociales y productivos, así como también sobre las demás tecnologías de punta y la investigación, contribuyendo a elevar la productividad total de los factores [1].

A nivel país, se observa en los últimos años un conjunto de acciones promovidas por el estado, las empresas y las universidades, de las cuales puede inferirse la relevancia del sector TIC en los planes estratégicos de desarrollo. En [2] se presenta una reseña de las acciones orientadas a la promoción de la industria del software, en el contexto nacional y en la región NEA.

Por tanto, formar recursos humanos en las áreas relacionadas con TIC con capacidad de innovación para aplicarlo en desarrollos específicos, es fundamental para el desarrollo de los países [3].

Por otra parte, el sector de Software y Servicios Informáticos (SSI) en nuestro país ha tenido un importante crecimiento en los últimos diez años, y se constituye como uno de los principales demandantes de empleo

$(*)$ Departamento de Informática. Facultad de Ciencias Exactas y Naturales y Agrimensura - Universidad Nacional del Nordeste, Corrientes, Argentina - gndapozo@exa.unne.edu.ar 
calificado. Sin embargo, la matrícula de las carreras universitarias no acompaña esta tendencia y resultan insuficientes los profesionales informáticos para el crecimiento de la industria. Ante esta problemática, diversos esfuerzos se encuentran en marcha para acercar a los jóvenes a las carreras vinculadas con las TIC. Entre ellas se destaca la iniciativa de la Fundación Dr. Manuel Sadosky, cuyo objetivo es favorecer la articulación entre el sistema científico tecnológico y la estructura productiva en todo lo referido a la temática de las TIC. Para ello lleva adelante programas tendientes a incrementar la cantidad de interesados por seguir carreras afines, y en particular propiciar la participación de las mujeres [4].

\section{Situación en la UNNE}

En la tabla 1 se muestra la evolución de la cantidad de alumnos inscriptos en la carrera Licenciatura en Sistemas de Información (LSI) de la UNNE, por año académico y sexo, desde el año 2000 al 2014. Se puede observar una marcada tendencia decreciente en el ingreso y una preponderancia de varones, más notoria en los últimos 6 años. Esta problemática se ve agravada por índices desfavorables de desgranamiento, deserción y lento avance en la carrera, que influye en el bajo número de egresados, un promedio de 40 alumnos por año ${ }^{1}$.

\begin{tabular}{|c|c|c|c|c|c|}
\hline Año & Varones & Mujeres & Total & \%Varones & \%Mujeres \\
\hline $\mathbf{2 0 0 0}$ & 673 & 440 & 1113 & $60 \%$ & $40 \%$ \\
\hline $\mathbf{2 0 0 1}$ & 308 & 210 & 518 & $59 \%$ & $41 \%$ \\
\hline $\mathbf{2 0 0 2}$ & 383 & 240 & 623 & $61 \%$ & $39 \%$ \\
\hline $\mathbf{2 0 0 3}$ & 462 & 230 & 692 & $67 \%$ & $33 \%$ \\
\hline $\mathbf{2 0 0 4}$ & 350 & 151 & 501 & $70 \%$ & $30 \%$ \\
\hline $\mathbf{2 0 0 5}$ & 381 & 160 & 541 & $70 \%$ & $30 \%$ \\
\hline $\mathbf{2 0 0 6}$ & 313 & 143 & 456 & $69 \%$ & $31 \%$ \\
\hline $\mathbf{2 0 0 7}$ & 311 & 115 & 426 & $73 \%$ & $27 \%$ \\
\hline $\mathbf{2 0 0 8}$ & 290 & 96 & 386 & $75 \%$ & $25 \%$ \\
\hline $\mathbf{2 0 0 9}$ & 274 & 61 & 335 & $82 \%$ & $18 \%$ \\
\hline $\mathbf{2 0 1 0}$ & 289 & 66 & 355 & $81 \%$ & $19 \%$ \\
\hline $\mathbf{2 0 1 1}$ & 240 & 73 & 313 & $77 \%$ & $23 \%$ \\
\hline $\mathbf{2 0 1 2}$ & 266 & 36 & 302 & $88 \%$ & $12 \%$ \\
\hline $\mathbf{2 0 1 3}$ & 238 & 53 & 291 & $82 \%$ & $18 \%$ \\
\hline $\mathbf{2 0 1 4}$ & 169 & 41 & 210 & $80 \%$ & $20 \%$ \\
\hline
\end{tabular}

TABLA 1: Evolución de los inscriptos en la LSI

\footnotetext{
${ }^{1} \mathrm{http}: / /$ www.unne.edu.ar/conociendo/egresados.php
} 


\section{La cuestión de género}

Un caso particular de atención respecto a las vocaciones en TIC lo representan las mujeres. En la LSI se observa una tendencia decreciente del porcentaje de mujeres, llegando a un 12\% en el año 2012 (Tabla 1). Esta situación coincide con lo expuesto por Gil Juárez [5], Fernández y otros [6].

Diversos estudios muestran que cada vez se incorporan más mujeres a las universidades, pero también coinciden en señalar que el porcentaje de mujeres es claramente inferior en las carreras científico-tecnológicas, y aun cuando en los últimos años se habla de un muy lento aumento de mujeres en esta área, existe una excepción: las titulaciones informáticas. Las mujeres no sólo no han ido incorporándose a estos estudios, sino que en muchos casos en las últimas dos décadas desciende su presencia, siendo este un fenómeno de alcance mundial [5].

Sanz [7] intenta explicar la disminución de las mujeres en las carreras de Informática, señalando que la importancia y prestigio que la Informática adquirió en la década de los '90, propició la incorporación de las mujeres. Su estudio sugiere que cuando un campo es incipiente, las mujeres se animan a adentrarse en él porque aún carece de estereotipos de género, pero luego la Informática se fue volviendo una ciencia cada vez más matematizada y cercana a las ingenierías o "ciencias duras" lo que significa, en términos de género, más masculinizada.

Un estudio basado en una muestra del Programa para la Evaluación Internacional de Alumnos (PISA) señala que la inequidad de género en la educación científica es un problema transnacional, y que los estereotipos de género juegan un rol importante [8].
En nuestro país la Fundación Sadosky ha realizado un estudio sobre representaciones acerca de la Informática en escuelas secundarias, a fin de inferir causas sobre la baja presencia femenina en informática [9].

\section{Por qué motivo los jóvenes no eligen ca- rreras TIC?}

En nuestro país, la CESSI (Cámara de Software y Servicios Informáticos) realizó en el año 2006 un estudio que reporta algunas causas por las cuáles los jóvenes no eligen carreras TIC [10]. Entre las causas que se mencionan, se destacan:

- Los jóvenes tienden a asociar a los informáticos con la imagen del "nerd", personas aisladas, enfrascadas en la computadora.

- Imaginan a las carreras TIC como difíciles, en parte por deficiencias en formación matemática, en parte por prejuicio puro.

- No tienen modelos a seguir asociados a estas carreras, personas que los inspire a inclinarse por la disciplina.

- El cambio en la educación en computación, que ha puesto el foco en el manejo de herramientas de ofimática en lugar de programación de computadoras.

- Los jóvenes desconocen los beneficios del desarrollo profesional en carreras relacionadas (buenos sueldos, desempleo cero, posibilidad de teletrabajo, etc).

Atendiendo a esta situación, la LSI de la UNNE, decidió acompañar la iniciativa de la Fundación Sadosky participando del programa Vocaciones en TIC. Este programa tiene como objetivo principal despertar 
interés en los jóvenes para estudiar carreras vinculadas con las TIC, en forma más amena y desestructurada, mediante la programación de juegos y animaciones, siendo sus objetivos particulares los siguientes:

$>$ Acercar a los alumnos del nivel medio experiencias prácticas acerca de las actividades propias del quehacer del profesional Informático.

$>$ Incorporar las nociones básicas de programación mediante actividades lúdicas que permiten apoyar la estructura de conocimientos de la disciplina Informática.

Como parte de las actividades, además, se relevó información para determinar el perfil de los alumnos del nivel medio en relación a su formación y habilidades tecnológicas, su interés por la elección de carreras vinculadas con la Informática y los factores que influyen en esta elección, a fin de aportar información que contribuya al objetivo de acercar a los jóvenes a la Informática, como actividad profesional.

\section{Metodología}

Para cumplir con los objetivos previstos, se realizaron las siguientes acciones:

- Formalización de un acuerdo específico entre la Facultad de Ciencias Exactas de la UNNE y la Fundación Sadosky, para llevar adelante las actividades previstas en el programa Vocaciones en TIC, específicamente en el proyecto Visita a las Escuelas.

- Realización de una capacitación específica al equipo formado por 6 (seis) alumnos de la LSI, acompañados por un docente, para el dictado de los talleres de capacitación en el uso de la herramienta Alice (Lenguaje de programación educati- vo y abierto orientado a objetos, desarrollado por la universidad de Carnegie Mellon). Esta capacitación, además incluyó charlas sobre la importancia de la formación profesional en Informática, las características de la profesión y las posibilidades laborales, para que sean transmitidas a los alumnos del nivel medio, dado que en general estos alumnos desconocen las posibilidades de la formación en TIC.

- Planificación de las visitas a las escuelas, tomando contacto con sus directivos y los docentes referentes tecnológicos.

- Realización de los talleres de programación con la herramienta Alice para la creación de juegos y animaciones en cada una de las escuelas seleccionadas, a cargo de alumnos de la LSI. Cada taller se desarrolló en 2 encuentros, totalizando 6 hs. reloj.

- Al finalizar el taller en cada escuela, se brindó una charla a los alumnos explicando qué significa trabajar y estudiar en el sector TIC. Además, se alentó a los alumnos a participar en la competencia Desafío Dale Aceptar, proyecto que forma parte también del programa Vocaciones en TIC y la competencia "El mundo animado de Alice", organizado por FaCENA para los alumnos que recibieron la capacitación.

- En cada escuela se solicitó a los alumnos completar un cuestionario (cuyo diseño se basó en el especificado en [11]), orientado a obtener información acerca de la formación de los alumnos en herramientas específicas de Informática, sus habilidades tecnológicas, su interés en acceder a una formación universitaria, y si la carrera estaría vinculada con la Informática, y qué factores influyen en la elección de la misma.

- Los datos fueron codificados e in- 
troducidos en una planilla de cálculo a partir de la cual se obtuvieron los resultados.

\section{Resultados}

Se visitaron 8 escuelas ( 7 correspondientes a Corrientes Capital y 1 a una localidad situada en el interior de la provincia), en el periodo comprendido entre el 13 de agosto al 30 de septiembre de 2.013. Un total de 297 alumnos de los últimos años de estas escuelas respondieron el cuestionario.

De las escuelas que participaron de la experiencia, 1 es de gestión privada, 3 son semiprivadas y 4 corresponden a escuelas públicas.

A continuación se muestran los resultados más significativos:
1. ¿Recibió capacitación formal en temas de Informática/Computación?: 194 (65\%) de los 297 alumnos expresó que recibió capacitación formal.

2. ¿Sobre cuáles de estos temas informáticos (programación, ofimática, diseño web, redes, otros) se capacitó formalmente?

La tabla 2 muestra que en la mayoría de las escuelas, los porcentajes más altos corresponden a herramientas de ofimática. Solamente la escuela técnica, que tiene una orientación en TIC, tiene un porcentaje significativo de programación. Así también se observa que el Instituto de Música sobresale en capacitación en diseño web (71\%).

\begin{tabular}{|l|r|r|r|r|r|}
\hline \multicolumn{1}{|c|}{ Escuela } & Programación & Ofimática & Diseño web & Inst. Redes & Otros \\
\hline Escuela Comercial & $50 \%$ & $61 \%$ & $22 \%$ & $28 \%$ & $17 \%$ \\
\hline Instituto de Música & $29 \%$ & $100 \%$ & $71 \%$ & $0 \%$ & $7 \%$ \\
\hline Colegio Informático & $45 \%$ & $71 \%$ & $26 \%$ & $11 \%$ & $24 \%$ \\
\hline Escuela Leloir & $50 \%$ & $100 \%$ & $17 \%$ & $25 \%$ & $8 \%$ \\
\hline Instituto PIO XI & $50 \%$ & $50 \%$ & $50 \%$ & $13 \%$ & $13 \%$ \\
\hline Esc Técnica U.O.C.R.A & $77 \%$ & $73 \%$ & $31 \%$ & $23 \%$ & $46 \%$ \\
\hline Instituto Roubineau & $48 \%$ & $57 \%$ & $13 \%$ & $17 \%$ & $22 \%$ \\
\hline Escuela Normal SL & $30 \%$ & $74 \%$ & $13 \%$ & $0 \%$ & $17 \%$ \\
\hline
\end{tabular}

TABLA 2: Capacitación en herramientas informáticas

Esta información confirma que la escuela secundaria no brinda, en general, contenidos y habilidades vinculados con la programación, que es la esencia de la disciplina Informática. Formar en herramientas de ofimática capacita a los alumnos en el papel de "usuarios" de las tecnologías, y no en el rol de "creadores" de tecnologías.

3. ¿En qué lugar recibió la capacitación?

De los 194 alumnos con capacitación formal, el 81\% recibió la capacitación en la escuela, un $15 \%$ en institutos externos al colegio y un $11 \%$ en otros lugares.

4. ¿Cuáles de estas tareas realizó alguna vez por su cuenta?

Se definieron tareas que requieren determinadas habilidades tecnológicas que denotan un perfil inclinado hacia las TIC. En el gráfico 1 se muestran los resultados obtenidos. 


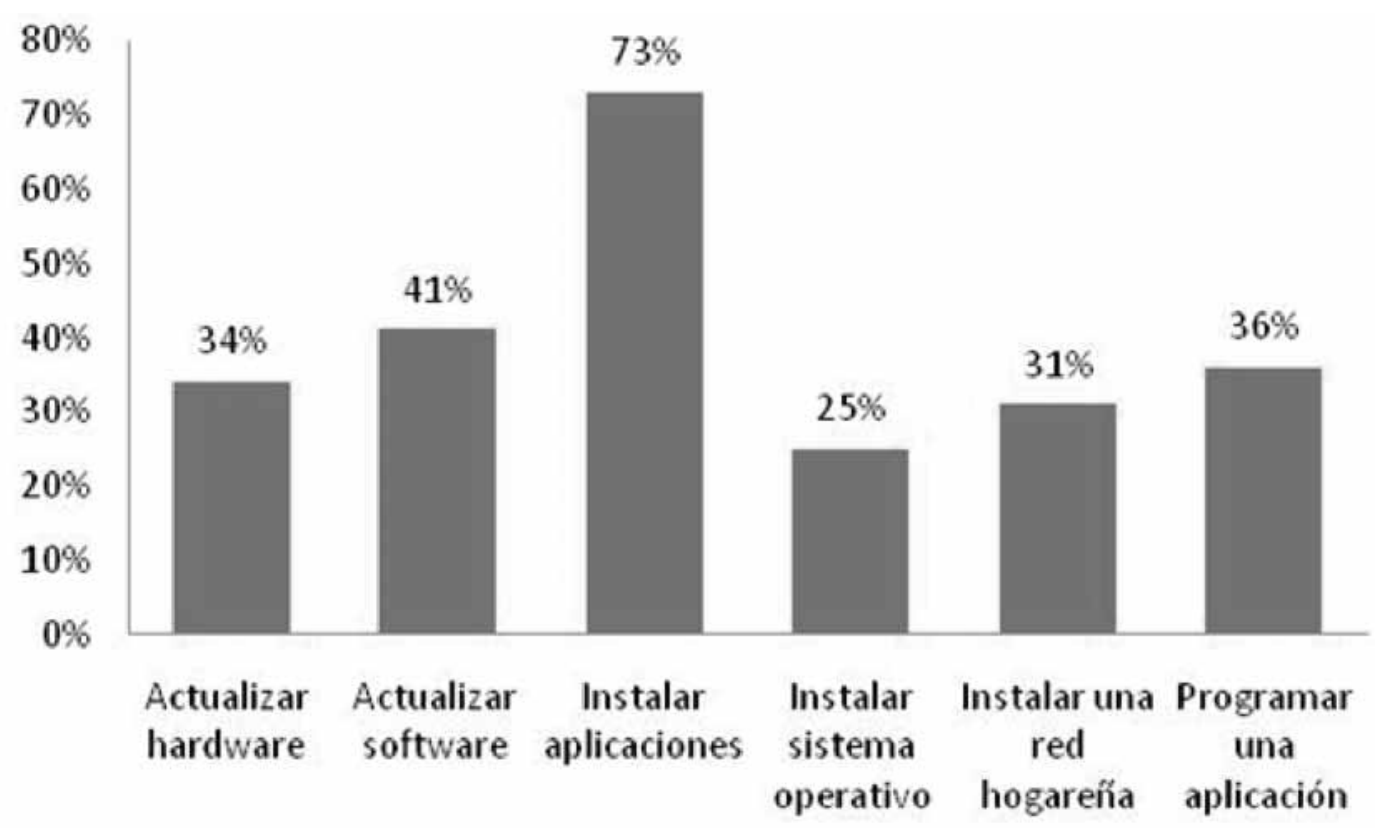

GRÁFICO 1: Habilidades tecnológicas

Se destaca que un $73 \%$ de los alumnos ha instalado aplicaciones alguna vez, el $31 \%$ ha instalado una red hogareña y el $25 \%$ ha instalado un sistema operativo. Estas cifras permiten inferir que instalar aplicaciones es una actividad bastante común para la totalidad de los alumnos pero no así instalar un sistema operativo o una red.

5. ¿Qué tan probable es que realice una carrera universitaria en el corto plazo?

Se definieron 5 categorías, que van gradualmente de Ninguna Posibilidad a Muy Probable. Se obtuvieron los siguientes porcentajes: Ninguna Posibilidad(13\%), Altamente Improbable (12\%), Algo Probable (35\%) y Muy Probable (39\%).

6. ¿Qué tan probable es que elija una carrera vinculada con la Informática?

Solo un 14\% (43 alumnos) manifestó un fuerte interés en iniciar una carrera vinculada con la Informática, un 38\% (113 alumnos) indicó que sería Algo Probable. Los que indicaron Ninguna posibilidad o Altamente Improbable constituyen el 46\% (135 alumnos). El alto porcentaje de Algo Probable podría indicar que la carrera es considerada como segunda opción, o que las ofertas de carreras de Informática no cubren totalmente las expectativas de estos alumnos.

Alumnos interesados en estudiar Informática

Para analizar las características de los alumnos que tienen interés en realizar una carrera relacionada con la Informática, se consideraron 3 categorías en función de su interés por seguir una carrera vinculada con la Informática:

1. Muy probable que sigan una carrera Informática.

2. Algo probable que sigan una carrera Informática 
3. No tienen interés en seguir una carrera Informática (abarca a los que contestaron Ninguna Posibilidad o Altamente Improbable).

Se analizó el comportamiento de la variable habilidades tecnológicas en relación a las categorías mencionadas. El gráfico 2 muestra que no existen diferencias significativas entre los alumnos que indicaron Algo Probable y los que No tienen interés en estudiar la carrera de Informática, excep- to en la tarea de Instalar programas, en la que se observan altos porcentajes en las 3 categorías. Esto seguramente está influido por la facilidad bastante extendida de instalar aplicaciones en los teléfonos inteligentes.

Se observa que en la categoría Muy Probable, las habilidades tecnológicas con mayor nivel de complejidad, como Instalar Sistema Operativo, Instalar Red y Programar, presentan porcentajes más altos.

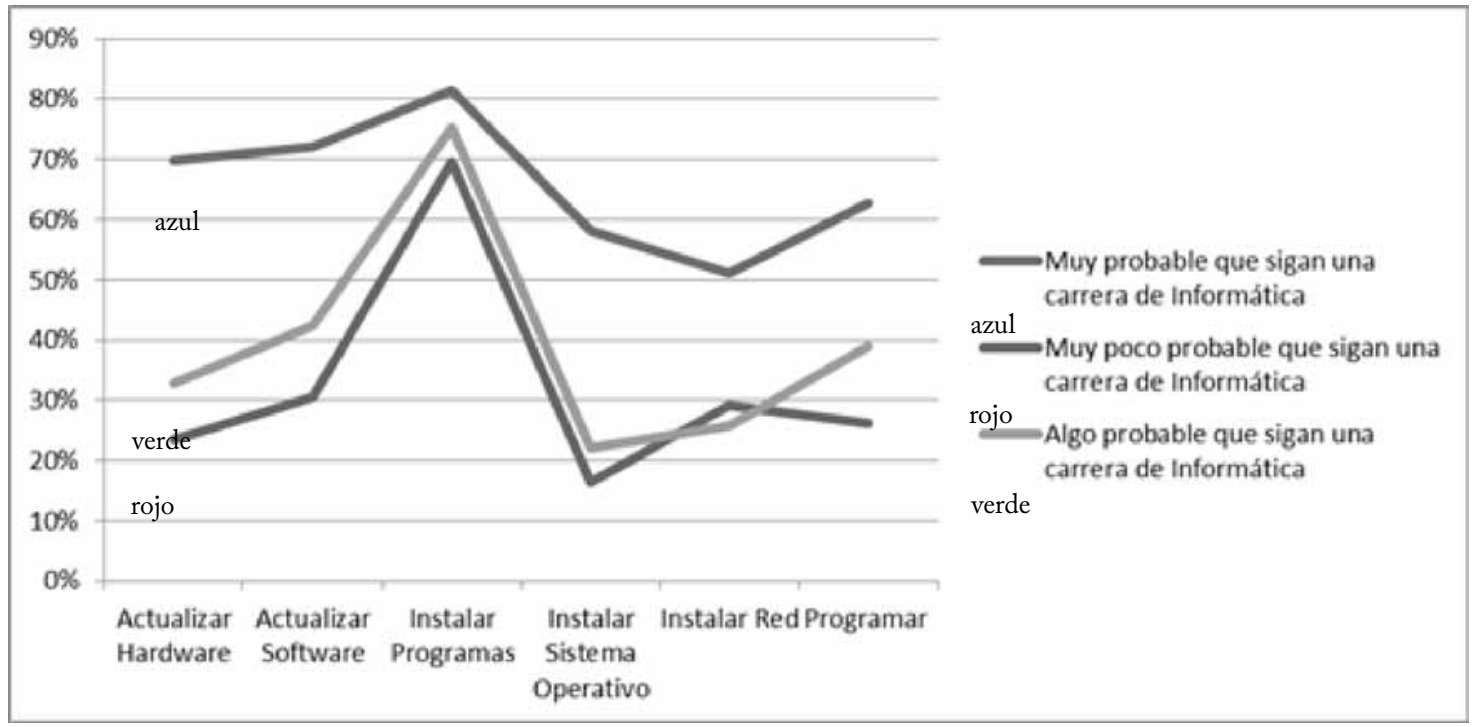

GRÁFICO 2: Categorías de probabilidad y habilidades tecnológicas

La inclinación hacia estas actividades representa una cierta predisposición o habilidad con la tecnología de computadoras, que se presentan con mayor frecuencia en los alumnos interesados en seguir carreras vinculadas con la Informática.

Sin embargo, conviene destacar que el desarrollo de estas habilidades tecnológicas difiere entre varones y mujeres. Para la categoría Muy Probable, el porcentaje de varones que realizan actividades específicas es mayor que el de las mujeres, más aún en aquellas actividades más vinculadas al hardware, tales como, Actualizar hardware, Instalar Sistema Operativo e Instalar Redes, tal como se muestra en el GRÁfICO 3.

\section{Factores influyentes en la elección de carrera}

Se presentó a los alumnos una lista de factores de posible influencia en su decisión de elegir una carrera vinculada con la Informática, y ellos debían señalar si la influencia era: A favor, En contra o Ninguna. Estos 


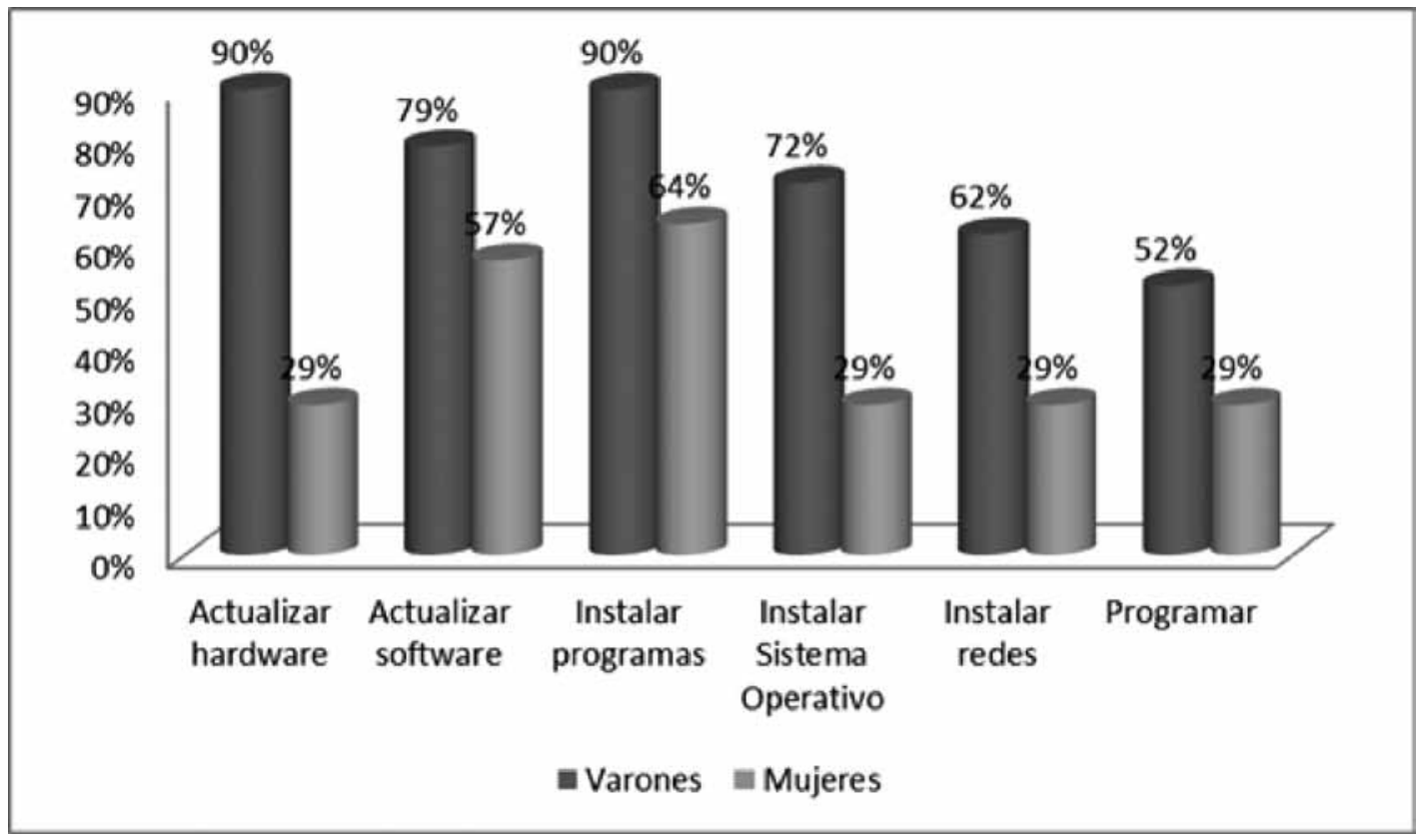

GRÁFICO 3: Actividades tecnológicas por género

factores son los siguientes:

- Mi conocimiento sobre lo que la carrera es

- La experiencia que tengo con la computadora campo

- Mi deseo de usar la informática en otro

- Conocer los salarios que se ganan en la actividad laboral

- Mi deseo de estar sentado enfrente de la computadora todo el día

- Mi interés en la programación de computadoras

- Mi interés en juegos de computadoras

- Mi interés en el hardware de computadoras

- Conozco a alguien en el campo de la Informática.
En el gráfico 4 se observa que en la categoría Muy Probable, los factores relevantes a la hora de inclinarse por la Informática son: Conocimiento sobre lo que la carrera es (81\%) y Experiencia que tengo con computadoras (81\%). Los siguientes factores a favor son: Interés en los juegos (74\%), Interés en el hardware (74\%) Interés en la Programación (72\%).

En tanto que en la categoría Algo Probable, se destacan: la Experiencia que tengo con computadoras (67\%) y Mi deseo de usar la Informática en otro campo (negocios, medicina,..) (63\%). Este último factor presenta porcentajes mayores a las otras categorías. Esta situación refleja una perspectiva particular de la Informática para los alumnos cuya elección fue Algo probable, que convendría profundizar.

En todos los casos, el factor con menor influencia fue: Mi deseo de estar sentado enfrente de la computadora todo el día. 


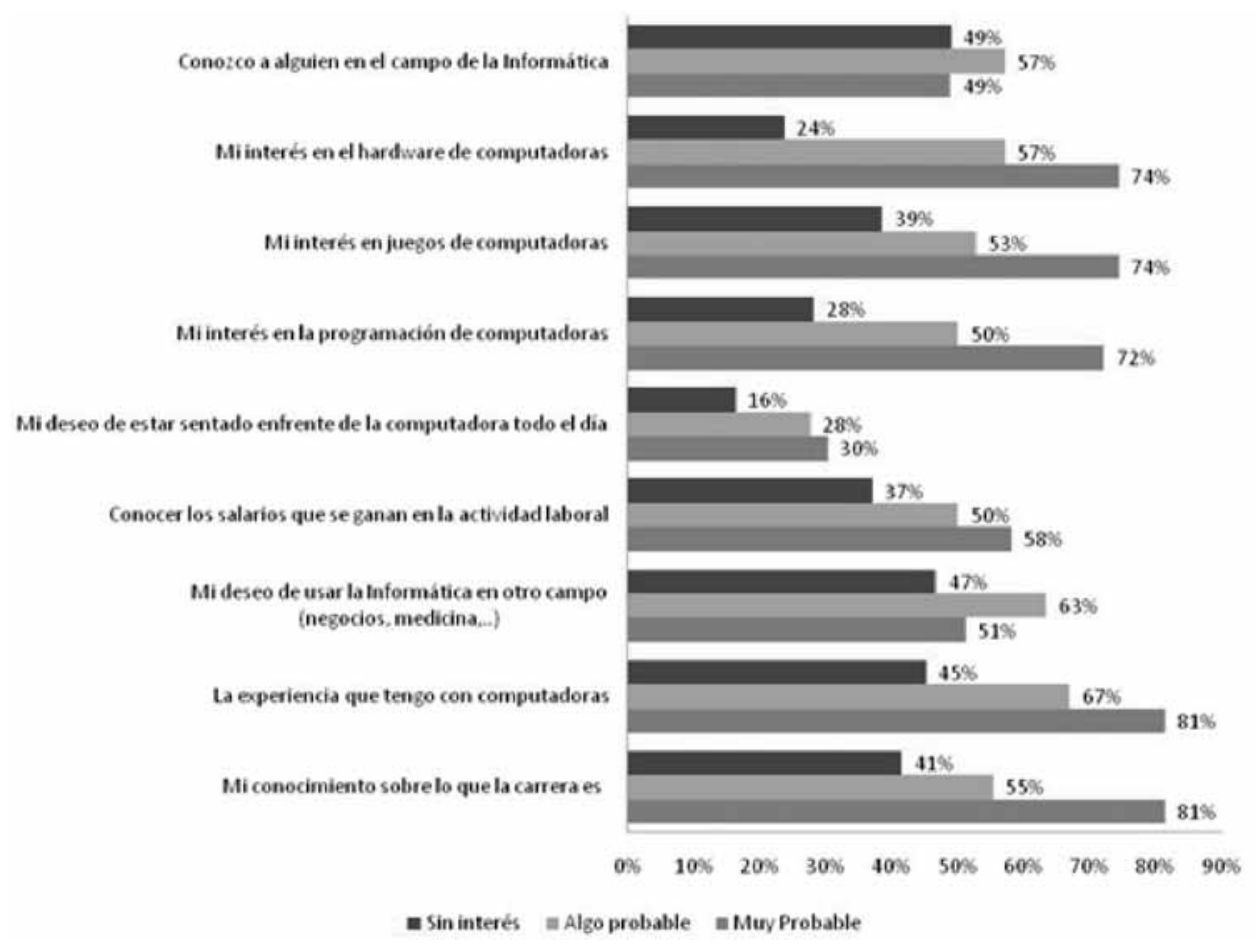

GRAFICO 4: Factores influyentes

\section{Conclusión}

De los resultados obtenidos se infiere que los alumnos interesados en seguir una carrera vinculada con la Informática se destacan por poseer mayores habilidades tecnológicas en relación con la computadora, siendo estas habilidades menores en el caso de las mujeres, y entre los factores que influyen positivamente en su elección, destacan: Conocimiento sobre lo que la carrera es, Experiencia que tengo con computadoras, Interés en los juegos, Interés en el hardware e Interés en la Programación.

Es más evidente el perfil de los varones que tienen una predisposición a seguir una carrera informática, pero no es así en el caso de las mujeres. Es necesario profundizar sobre sus intereses para que los esfuerzos orientados a promover vocaciones TIC sean inclusivos para ambos géneros y no aumente la brecha entre los mismos.

Este estudio de las características de los alumnos, fortalece la idea de que motivar tempranamente a los jóvenes en el conocimiento práctico de las tecnologías informáticas incrementará su interés por estudiar carreras TIC.

Por otra parte, de la experiencia de las visitas a las escuelas cabe destacar la motivación de los alumnos capacitadores de la LSI, quienes desempeñaron su tarea con un fuerte compromiso y espíritu de pertenencia, llevando a la práctica un excelente trabajo de equipo. Además, la oportunidad de vivenciar las distintas realidades de la educación secundaria, contribuirá a su formación ciudadana y al aporte de soluciones para superar situaciones de inequidad para el acceso a la educación universitaria. 


\section{Referencias}

[1] Ministerio de Ciencia y Tecnología (2009)."Boletín Estadístico Tecnológico”. N 2. http://www.mincyt.gov. ar/indicadores/banco_indicadores/publicaciones/bet_TIC_final.pdf

[2] Cuenca Pletsch, L.; Dapozo, G.; Greiner, C.; Estayno, M. (2012) “Vinculación Universidad-Empresa orientada a la promoción de la industria del software. Una experiencia de colaboración en la región NEA”. Revista del Núcleo de Estudios e Investigaciones en Educación Superior del MERCOSUR. ISSN 2313-9080. Vol. 1 (2012).

[3] RedUNCI (Red de Universidades Nacionales con Carreras en Informática). (2014) Formación de Recursos Humanos. Documento de trabajo.http:// redunci.info.unlp.edu.ar

[4] Fundación Dr. Manuel Sadosky de Investigación y Desarrollo en las Tecnologías de la Información y Comunicación. http://www.fundacionsadosky.org.ar/

[5] Gil-Juárez, A.; Feliu, J.; Vitores, A. (2012) "Género y TIC: en torno a la brecha digital de género". Revista Athenea Digital, 12(3), 3-9.

[6] Fernández, V.; Larraza, E.; Maritxalar, M.; Ruiz, T; Sarasola, K. (2006). “Ingeniería en Informática y Género. Un estudio cuantitativo". VI Congreso Iberoamericano de Ciencia, Tecnología y Género. Zaragoza. España.

[7] Sanz, V. (2008). Mujeres e Ingeniería Informática: El caso de la facultad de Informática de la UPM. ARBOR Ciencia, Pensamiento y Cultura, CLXXXIV 733. Pag. 905-915.

[8] Buccheria, G.; AbtGürbera, N.; Brühwiler, C. (2011). "The Impact of Gender on Interest in Science Topics and the Choice of Scientific and Technical Vocations". Disponible en:http://www.tandfonline.com/doi/abs/10.1080/0 9500693.2010.518643\#.U8kJVpR5N0s

[9] FundaciónSadosky. (2014) "Y las mujeres ¿dónde están?”. Disponible en: http://www.fundacionsadosky.org. ar/wp-content/uploads/2014/06/informe-mujeres-y-computacion-2013.pdf

[10] Benotti, L.: Echeveste, M.E.; Schapachnik, F. (2012), "Despertando Vocaciones en Computación mediante el uso de autómatas de chat". JAIIO 2012.

[11] Carter, L. (2006). "Why Students with an Apparent Aptitude for Computer Science Don't Choose to Major in Computer Science”. SIGCSE'06, March 1-5. 